\section{Laienreanimation auch unter funktionellem Aspekt von Vorteil}

Einen Herzstillstand überleben ist das Eine. Das Andere - und für die meisten Betroffenen mindestens ebenso wichtig - ist, ihre körperliche und geistige Funktionalität weitestgehend zu bewahren. Auch dafür ist offenbar das tatkräftige Einschreiten eines Ersthelfers ausschlaggebend, wie eine dänische Studie nun verdeutlicht. Denn hier war das Risiko für Betroffene nach einem Herzstillstand außerhalb einer Klinik, anoxische Hirnschäden zu erleiden oder in ein Pflegeheim eingewiesen zu werden, im Fall einer Laienreanimation um $30 \%$ geringer als für Überlebende, die keine solche Wiederbelebungsmaßnahme erfahren hatten. Noch deutlicher, nämlich um $40 \%$, sank das Risiko, wenn Ersthelfer stattdessen oder zusätzlich einen automatisierten externen Defibrillator (AED) eingesetzt hatten.

\section{Nur zwei von zehn Überlebenden müssen ins Pflegeheim}

Hoffnungsvoll stimmt zudem, wie viele der Überlebenden sich ein Jahr nach dem Ereignis in einem guten Zustand befanden: Bei über $80 \%$ der Überlebenden kam es nach einer Laienreanimation weder zu Hirnschädigungen noch zu einem Heimeintritt, ohne diese Maßnahmen war dies bei gut $70 \%$ der Fall. Drei von zehn Überlebenden, die keine Reanimation durch einen Ersthelfer erfahren hätten, seien ein Jahr später im Pflegeheim gewesen oder hätten Hirnschäden erlitten, verdeutlichte Dr. Kristian Kragholm von der Universitätsklinik in Aalborg /Dänemark die Ergebnisse auf dem ESC-Kongress 2015 in London. Nach einer Wiederbelebung durch einen Ersthelfer würde dies auf zwei von zehn Überlebenden zutreffen.

Insgesamt überlebten in Dänemark 2.469 von 32.883 Personen, die zwischen 2001 und 2011 einen Herzstillstand erlitten hatten, 30 Tage lang. Die Laienreanimation war dabei der einzige signifikante Faktor, der mit einem geringeren Risiko für eine Pflegeheimeinweisung oder Hirnschädigung einherging.

Dieser Befund unterstreiche die Bedeutsamkeit von Wiederbelebungsmaßnahmen durch Laien sowie die Nutzung von AEDs in Notfallsituationen, betonte Kragholm. Denn dadurch würden sich nicht nur die Überlebenschancen nach einem Herzstillstand erhöhen, sondern auch die Wahrscheinlichkeit, dass der Betroffene seine physische und mentale Funktionalität bewahrt. Veronika Schlimpert

\section{Vorhofflimmern-Ablation: Weder ATP noch Antiarrhythmika reduzieren Rezidivrate}

\begin{abstract}
Nicht alle Studien enden wie erhofft. So ergab eine große japanische Studie, dass weder Antiarrhythmika noch ein ATP-Test-gesteuertes Vorgehen bei der Katheterablation von Vorhofflimmern die Rezidivraten senken können.
\end{abstract}

$A^{n}$ der KPAF („Kansai Plus Atrial Fibrillation")-Studie nahmen 2.120 Patienten teil, die sich wegen Vorhofflimmerns zum ersten Mal einer Katheterablation mit Pulmonalvenenisolation (PVI) unterzogen. Es handelte sich um eine Studie mit doppelter Randomisierung. In dem UNDER-ATP genannten ersten Teil der Studie wurde bei der Hälfte der Patienten nach der initialen PVI ein ATP-Test durchgeführt, bei der anderen Hälfte nicht. Mit dem ATP-Test wurde versucht, eventuell noch nicht abladierte Leitungsbahnen zu demaskieren. Diese wurden dann gezielt beseitigt, um Rezidive zu verhindern.

Für den zweiten, EAST-AF genannten Teil der Studie wurden sämtliche Patienten erneut randomisiert. Sie erhielten entweder für 90 Tage nach der Ablation antiarrhythmische Medikamente oder nicht. Eingesetzt wurden unterschied-

lichste Substanzen, am häufigsten Flecainid, Pilsicainid und Cibenzolin. Auch hier war die Intention, durch diese temporäre Behandlung langfristig Rezidive zu reduzieren.

\section{Beide Substudien enttäuschen}

In beiden Substudien gelang es aber letztlich nicht, die Rate der Patienten mit erneuten entweder behandlungsbedürftigen oder länger als 30 Sekunden andauernden Vorhof-Tachyarrhythmien zu senken. In der UNDER-ATP-Substudie hatten bei ATP-gesteuerter PVI $68,7 \%$ der Patienten nach Ablauf eines Jahres keine erneuten atrialen Tachyarrhythmien. In der konventionell therapierten Gruppe waren es kaum weniger, nämlich $67,1 \%(\mathrm{p}=0,19)$.

In der EAST-AF-Substudie sah es ähnlich aus: $69,5 \%$ der mit Antiarrhythmika und $67,8 \%$ der konventionell

behandelten Patienten waren nach einem Jahr

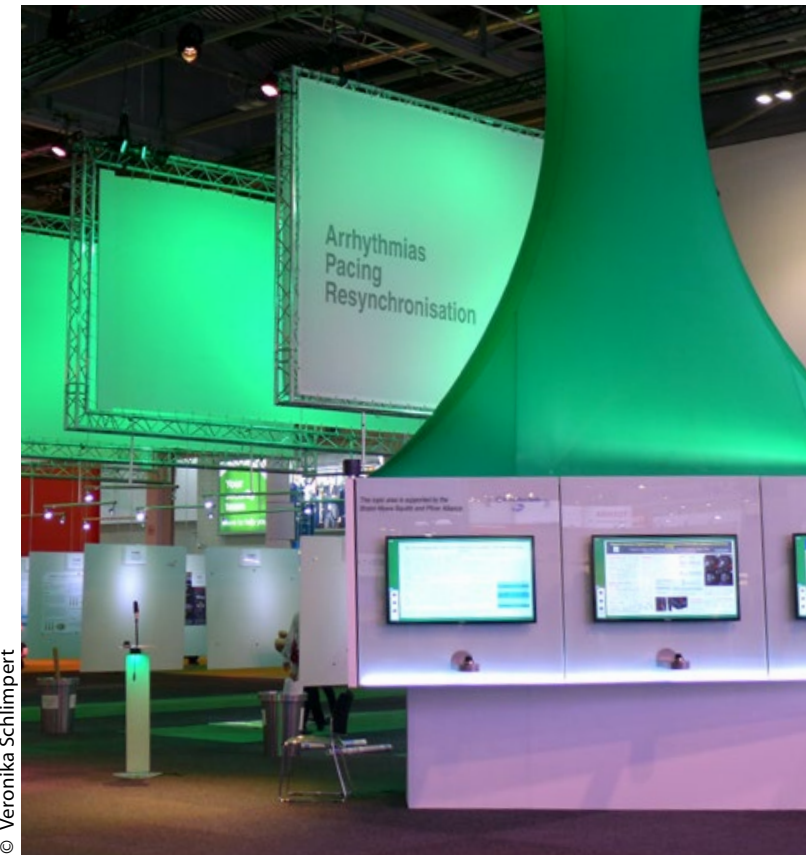

Neue Therapiestrategien gegen Arrhythmien waren auch in den ESC-Posterpräsentationen ein großes Thema. noch ohne erneute Vorhoftachykardien $(\mathrm{p}=0,38)$.

In dieser Auswertung wurden die ersten drei Monate, in denen antiarrhythmisch therapiert wurde, nicht mitgezählt, da es ja um die Langzeitrezidive ging. Wurden die ersten drei Monate separat ausgewertet, schnitt die Gruppe mit Antiarrhythmika-Therapie mit einer Rezidivrate von $41 \%$ erwartungsgemäß etwas besser ab als die Kontrollgruppe, wo $47,9 \%$ der Patienten Rezidive hatten.

Philipp Grätzel 